\title{
Decomposition of analytic measures on groups and measure spaces
}

\author{
by
}

\author{
Nakhlé Asmar and Stephen Montgomery-Smith (Columbia, MO) \\ Dedicated to the memory of Edwin Hewitt
}

\begin{abstract}
We consider an arbitrary locally compact abelian group $G$, with an ordered dual group $\Gamma$, acting on a space of measures. Under suitable conditions, we define the notion of analytic measures using the representation of $G$ and the order on $\Gamma$. Our goal is to study analytic measures by applying a new transference principle for subspaces of measures, along with results from probability and Littlewood-Paley theory. As a consequence, we derive new properties of analytic measures as well as extensions of previous work of Helson and Lowdenslager, de Leeuw and Glicksberg, and Forelli.
\end{abstract}

1. Introduction. This paper is essentially providing a new approach to generalizations of the F. \& M. Riesz Theorems, for example, such results as that of Helson and Lowdenslager $[15,16]$. They showed that if $G$ is a compact abelian group with ordered dual, and if $\mu$ is an analytic measure (that is, its Fourier transform is supported on the positive elements of the dual), then it follows that the singular and absolutely continuous parts (with respect to the Haar measure) are also analytic.

Another direction is that provided by Forelli [11] (itself a generalization of the result of de Leeuw and Glicksberg [21]), where one has an action of the real numbers $\mathbb{R}$ acting on a locally compact topological space $\Omega$, and a Baire measure $\mu$ on $\Omega$ that is analytic (in a sense that we make precise below) with respect to the action. Then again, the singular and absolutely continuous parts of $\mu$ (with respect to any so-called quasi-invariant measure) are also analytic.

Indeed, common generalizations of both these ideas have been provided, for example, by Yamaguchi [23], considering the action of any locally compact abelian group with ordered dual, on a locally compact topological space.

2000 Mathematics Subject Classification: 43A17, 43A32.

Key words and phrases: orders, transference, measure space, sup path attaining, F. \& M. Riesz Theorem. 
For more generalizations we refer the reader to Hewitt, Koshi, and Takahashi [18].

In the paper [4], a new approach to proving these kinds of results was given, providing a transference principle for spaces of measures. In that paper, the action was from a locally compact abelian group into a space of isomorphisms on the space of measures of a $\sigma$-algebra. A primary requirement that the action had to satisfy was what was called sup path attaining, a property that was satisfied, for example, by the setting of Forelli (Baire measures on a locally compact topological space). Using this transference principle, the authors were able to give an extension and a new proof of Forelli's result. This was obtained by using a Littlewood-Paley decomposition of an analytic measure.

In this paper we wish to continue this process, applying this same transference principle to provide the common generalizations of the results of Forelli and Helson and Lowdenslager. What we provide is essentially a decomposition of an analytic measure as a sum of martingale differences with respect to a filtration defined by the order. For each martingale difference, the action of the group can be described precisely by a certain action of the group of real numbers, and so we can appeal to the results of [4].

In this way, we can reach the following generalization (see Theorem 6.4 below): if $\mathcal{P}$ is any bounded operator on the space of measures that commutes with the action (as does, for example, taking the singular part), and if $\mu$ is an analytic measure, then $\mathcal{P} \mu$ is also an analytic measure.

In the remainder of the introduction, we establish our notation, including the notion of sup path attaining, and recall the transference principle from [4]. In Section 2, we describe orders on locally compact abelian groups, including the extension of Hahn's Embedding Theorem provided in [1]. In Section 3, we define the notions of analyticity. This somewhat technical section continues into Section 4, which examines the role of homomorphism with respect to analyticity. The technical results basically provide proofs of what is believable, and so may be skipped on first reading. It will be seen that the concept of sup path attaining comes up again and again, and may be seen to be an integral part of all our proofs.

In Section 5, we are ready to present the decomposition of analytic measures. This depends heavily on transference of martingale inequalities of Burkholder and Garling, and then using the fact that weakly unconditionally summing series are unconditionally summing in norm for any series in a space of measures [5]. In Section 6, we then give applications of this decomposition, giving the generalizations that we alluded to above.

Throughout, $G$ denotes a locally compact abelian group with dual group $\Gamma$. The symbols $\mathbb{Z}, \mathbb{R}$ and $\mathbb{C}$ denote the integers, the real and the complex numbers, respectively. If $A$ is a set, we denote the indicator function of $A$ 
by $1_{A}$. For $1 \leq p<\infty$, the space of Haar measurable functions $f$ on $G$ with $\int_{G}|f|^{p} d x<\infty$ is denoted by $L^{p}(G)$. The space of essentially bounded functions on $G$ is denoted by $L^{\infty}(G)$. The expressions "locally null" and "locally almost everywhere" have the same meanings as in $[19$, Definition (11.26)].

Let $\mathcal{C}_{0}(G)$ denote the Banach space of continuous functions on $G$ vanishing at infinity. The space of all complex regular Borel measures on $G$, denoted by $M(G)$, consists of all complex measures arising from bounded linear functionals on $\mathcal{C}_{0}(G)$.

Let $(\Omega, \Sigma)$ denote a measurable space, where $\Omega$ is a set and $\Sigma$ is a $\sigma$-algebra of subsets of $\Omega$. Let $M(\Sigma)$ denote the Banach space of complex measures on $\Sigma$ with the total variation norm, and let $\mathcal{L}^{\infty}(\Sigma)$ denote the space of measurable bounded functions on $\Omega$.

Let $T: t \mapsto T_{t}$ denote a representation of $G$ by isomorphisms of $M(\Sigma)$. We suppose that $T$ is uniformly bounded, i.e., there is a positive constant $c$ such that for all $t \in G$, we have

$$
\left\|T_{t}\right\| \leq c .
$$

Definition 1.1. A measure $\mu \in M(\Sigma)$ is called weakly measurable (in symbols, $\left.\mu \in \mathcal{M}_{T}(\Sigma)\right)$ if for every $A \in \Sigma$ the mapping $t \mapsto T_{t} \mu(A)$ is Borel measurable on $G$.

Given a measure $\mu \in \mathcal{M}_{T}(\Sigma)$ and a Borel measure $\nu \in M(G)$, we define the "convolution" $\nu *_{T} \mu$ on $\Sigma$ by

$$
\nu *_{T} \mu(A)=\int_{G} T_{-t} \mu(A) d \nu(t)
$$

for all $A \in \Sigma$.

We assume throughout this paper that the representation $T$ commutes with the convolution (2) in the following sense: for each $t \in G$,

$$
T_{t}\left(\nu *_{T} \mu\right)=\nu *_{T}\left(T_{t} \mu\right) .
$$

Condition (3) holds if, for example, for all $t \in G$, the adjoint of $T_{t}$ maps $\mathcal{L}^{\infty}(\Sigma)$ into itself. In symbols,

$$
T_{t}^{*}: \mathcal{L}^{\infty}(\Sigma) \rightarrow \mathcal{L}^{\infty}(\Sigma)
$$

For proofs we refer the reader to [2]. Using (1) and (3), it can be shown that $\nu *_{T} \mu$ is a measure in $\mathcal{M}_{T}(\Sigma)$,

$$
\left\|\nu *_{T} \mu\right\| \leq c\|\nu\| \cdot\|\mu\|
$$

where $c$ is as in (1), and

$$
\sigma *_{T}\left(\nu *_{T} \mu\right)=(\sigma * \nu) *_{T} \mu
$$

for all $\sigma, \nu \in M(G)$ and $\mu \in \mathcal{M}_{T}(\Sigma)$ (see [2]). 
Definition 1.2. A representation $T=\left(T_{t}\right)_{t \in G}$ of a locally compact abelian group $G$ in $M(\Sigma)$ is said to be sup path attaining if it is uniformly bounded, has property (3), and there is a constant $C$ such that for every weakly measurable $\mu \in \mathcal{M}_{T}(\Sigma)$ we have

$$
\|\mu\| \leq C \sup \left\{\operatorname{esssup}_{t \in G}\left|\int_{\Omega} h d\left(T_{t} \mu\right)\right|: h \in \mathcal{L}^{\infty}(\Sigma),\|h\|_{\infty} \leq 1\right\} .
$$

The fact that the mapping $t \mapsto \int_{\Omega} h d\left(T_{t} \mu\right)$ is measurable is a simple consequence of the measurability of the mapping $t \mapsto T_{t} \mu(A)$ for every $A \in \Sigma$.

In [4] were provided many examples of sup path attaining representations. Rather than give this same list again, we give a couple of examples of particular interest.

EXAMPLE 1.3. (a) (This is the setting of Forelli's Theorem.) Let $G$ be a locally compact abelian group, and $\Omega$ be a locally compact topological space. Suppose that $\left(T_{t}\right)_{t \in G}$ is a group of homeomorphisms of $\Omega$ onto itself such that the mapping $(t, \omega) \mapsto T_{t} \omega$ is jointly continuous. Then the space of Baire measures on $\Omega$, that is, the minimal $\sigma$-algebra such that compactly supported continuous functions are measurable, is sup path attaining under the action $T_{t} \mu(A)=\mu\left(T_{t}(A)\right.$ ), where $T_{t}(A)=\left\{T_{t} \omega: \omega \in A\right\}$. (Note that all Baire measures are weakly measurable.)

(b) Suppose that $G_{1}$ and $G_{2}$ are locally compact abelian groups and that $\phi: G_{2} \rightarrow G_{1}$ is a continuous homomorphism. Define an action of $G_{2}$ on $M\left(G_{1}\right)$ (the regular Borel measures on $G_{1}$ ) by translation by $\phi$. Hence, for $x \in G_{2}, \mu \in M\left(G_{1}\right)$, and any Borel $A \subset G_{1}$, let $T_{x} \mu(A)=\mu(A+\phi(x))$. Then every $\mu \in M\left(G_{1}\right)$ is weakly measurable, and the representation is sup path attaining with constants $c=1$ and $C=1$.

Proposition 1.4. Suppose that $T$ is sup path attaining and $\mu$ is weakly measurable such that for every $A \in \Sigma$ we have

$$
T_{t} \mu(A)=0
$$

for locally almost all $t \in G$. Then $\mu=0$.

The proof is immediate (see [2]).

We now recall some basic definitions from spectral theory.

If $I$ is an ideal in $L^{1}(G)$, let

$$
Z(I)=\bigcap_{f \in I}\{\chi \in \Gamma: \widehat{f}(\chi)=0\} .
$$

The set $Z(I)$ is called the zero set of $I$. For a weakly measurable $\mu \in M(\Sigma)$, let

$$
\mathcal{I}(\mu)=\left\{f \in L^{1}(G): f *_{T} \mu=0\right\} .
$$


When we need to be specific about the representation, we will use the symbol $\mathcal{I}_{T}(\mu)$ instead of $\mathcal{I}(\mu)$.

From properties of the convolution $*_{T}$, it is straightforward to show that $\mathcal{I}(\mu)$ is a closed ideal in $L^{1}(G)$.

Definition 1.5. The $T$-spectrum of a weakly measurable $\mu \in \mathcal{M}_{T}(\Sigma)$ is defined by

$$
\operatorname{spec}_{T} \mu=\bigcap_{f \in \mathcal{I}(\mu)}\{\chi \in \Gamma: \widehat{f}(\chi)=0\}=Z(\mathcal{I}(\mu)) .
$$

If $S \subset \Gamma$, let

$$
L_{S}^{1}=L_{S}^{1}(G)=\left\{f \in L^{1}(G): \widehat{f}=0 \text { outside of } S\right\} .
$$

In order to state the main transference result, we introduce one more definition.

Definition 1.6. A subset $S \subset \Gamma$ is a $\mathcal{T}$-set if, given any compact set $K \subset S$, each neighborhood of $0 \in \Gamma$ contains a nonempty open set $W$ such that $W+K \subset S$.

EXAMPLE 1.7. (a) If $\Gamma$ is a locally compact abelian group, then any open subset of $\Gamma$ is a $\mathcal{T}$-set. In particular, if $\Gamma$ is discrete then every subset of $\Gamma$ is a $\mathcal{T}$-set.

(b) The set $[a, \infty)$ is a $\mathcal{T}$-subset of $\mathbb{R}$ for all $a \in \mathbb{R}$.

(c) Let $a \in \mathbb{R}$ and $\psi: \Gamma \rightarrow \mathbb{R}$ be a continuous homomorphism. Then $S=\psi^{-1}([a, \infty))$ is a $\mathcal{T}$-set.

(d) Let $\Gamma=\mathbb{R}^{2}$ and $S=\left\{(x, y): y^{2} \leq x\right\}$. Then $S$ is a $\mathcal{T}$-subset of $\mathbb{R}^{2}$ such that there is no nonempty open set $W \subset \mathbb{R}^{2}$ such that $W+S \subset S$.

The main result of [4] is the following transference theorem.

THEOREM 1.8. Let $T$ be a sup path attaining representation of a locally compact abelian group $G$ by isomorphisms of $M(\Sigma)$ and let $S$ be a $\mathcal{T}$-subset of $\Gamma$. Suppose that $\nu$ is a measure in $M(G)$ such that

$$
\|\nu * f\|_{1} \leq\|f\|_{1}
$$

for all $f$ in $L_{S}^{1}(G)$. Then for every weakly measurable $\mu \in M(\Sigma)$ with $\operatorname{spec}_{T} \mu \subset S$ we have

$$
\left\|\nu *_{T} \mu\right\| \leq c^{3} C\|\mu\|,
$$

where $c$ is as in (1) and $C$ is as in (7).

2. Orders on locally compact abelian groups. An order $P$ on $\Gamma$ is a subset that satisfies the three axioms: $P+P \subset P ; P \cup(-P)=\Gamma$; and $P \cap(-P)=\{0\}$. We recall from [1] the following property of orders.

ThEOREM 2.1. Let $P$ be a measurable order on $\Gamma$. There are a totally ordered set $\Pi$ with largest element $\alpha_{0} ;$ a chain $\left\{C_{\alpha}\right\}_{\alpha \in \Pi}$ of subgroups of $\Gamma$; 


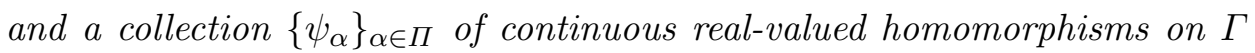
such that:

(i) for each $\alpha \in \Pi, C_{\alpha}$ is an open subgroup of $\Gamma$,

(ii) $C_{\alpha} \subset C_{\beta}$ if $\alpha>\beta$.

Let $D_{\alpha}=\left\{\chi \in C_{\alpha}: \psi_{\alpha}(\chi)=0\right\}$. Then, for every $\alpha \in \Pi$,

(iii) $\psi_{\alpha}(\chi)>0$ for every $\chi \in P \cap\left(C_{\alpha} \backslash D_{\alpha}\right)$,

(iv) $\psi_{\alpha}(\chi)<0$ for every $\chi \in(-P) \cap\left(C_{\alpha} \backslash D_{\alpha}\right)$,

(v) when $\Gamma$ is discrete, $C_{\alpha_{0}}=\{0\}$; and when $\Gamma$ is not discrete, $D_{\alpha_{0}}$ has empty interior and is locally null.

When $\Gamma$ is discrete, Theorem 2.1 can be deduced from the proof of Hahn's Embedding Theorem for orders (see [12, Theorem 16, p. 59]). The general case treated in Theorem 2.1 accounts for the measure theoretic aspect of orders. The proof is based on the study of orders of Hewitt and Koshi [17].

For $\alpha \in \Pi$ with $\alpha \neq \alpha_{0}$, let

$$
\begin{aligned}
S_{\alpha} \equiv P \cap\left(C_{\alpha} \backslash D_{\alpha}\right) & =\left\{\chi \in C_{\alpha} \backslash D_{\alpha}: \psi_{\alpha}(\chi) \geq 0\right\} \\
& =\left\{\chi \in C_{\alpha}: \psi_{\alpha}(\chi)>0\right\} .
\end{aligned}
$$

For $\alpha=\alpha_{0}$, set

$$
S_{\alpha_{0}}=\left\{\chi \in C_{\alpha_{0}}: \psi_{\alpha_{0}}(\chi) \geq 0\right\} .
$$

Note that when $\Gamma$ is discrete, $C_{\alpha_{0}}=\{0\}$, and so $S_{\alpha_{0}}=\{0\}$ in this case.

If $A$ is a subset of a topological space, we will use $\bar{A}$ and $A^{\circ}$ to denote the closure and the interior of $A$, respectively.

REMARKS 2.2. (a) It is a classical fact that a group $\Gamma$ can be ordered if and only if it is torsion-free. Also, an order on $\Gamma$ is any maximal positively linearly independent set. Thus, orders abound in torsion-free abelian groups, as they can be constructed using Zorn's Lemma to obtain a maximal positively linearly independent set. (See [17, Section 2].) However, if we ask for measurable orders, then we are restricted in many ways in the choices of $P$ and also the topology on $\Gamma$. As shown in [17], any measurable order on $\Gamma$ has nonempty interior. Thus, for example, while there are infinitely many orders on $\mathbb{R}$, only two are Lebesgue measurable: $P=[0, \infty[$, and $P=]-\infty, 0]$. It is also shown in [17, Theorem (3.2)] that any order on an infinite compact torsion-free abelian group is non-Haar measurable. This effectively shows that if $\Gamma$ contains a Haar measurable order $P$, and we use the structure theorem for locally compact abelian groups to write $\Gamma$ as $\mathbb{R}^{a} \times \Delta$, where $\Delta$ contains a compact open subgroup [19, Theorem (24.30)], then either $a$ is a positive integer, or $\Gamma$ is discrete. (See [1].) 
(b) The subgroups $\left(C_{\alpha}\right)$ are characterized as being the principal convex subgroups in $\Gamma$ and for each $\alpha \in \Pi$, we have

$$
D_{\alpha}=\bigcup_{\beta>\alpha} C_{\beta}
$$

Consequently, we have $C_{\alpha} \subset D_{\beta}$ if $\beta<\alpha$. By construction, the sets $C_{\alpha}$ are open. For $\alpha<\alpha_{0}$, the subgroup $D_{\alpha}$ has nonempty interior, since it contains $C_{\beta}$, with $\alpha<\beta$. Hence for $\alpha \neq \alpha_{0}, D_{\alpha}$ is open and closed. Consequently, for $\alpha \neq \alpha_{0}, C_{\alpha} \backslash D_{\alpha}$ is open and closed.

(c) Let $\psi: \Gamma_{1} \rightarrow \Gamma_{2}$ be a continuous homomorphism between two ordered groups. We say that $\psi$ is order preserving if $\psi\left(P_{1}\right) \subset P_{2}$. Consequently, if $\psi$ is continuous and order preserving, then $\psi\left(\bar{P}_{1}\right) \subset \bar{P}_{2}$.

For each $\alpha \in \Pi$, let $\pi_{\alpha}$ denote the quotient homomorphism $\Gamma \rightarrow \Gamma / C_{\alpha}$. Because $C_{\alpha}$ is a principal subgroup, we can define an order on $\Gamma / C_{\alpha}$ by setting $\psi_{\alpha}(\chi) \geq 0 \Leftrightarrow \chi \geq 0$. Moreover, the principal convex subgroups in $\Gamma / C_{\alpha}$ are precisely the images by $\pi_{\alpha}$ of the principal convex subgroups of $\Gamma$ containing $C_{\alpha}$. (See $[1$, Section 2].)

We end this section with a useful property of orders.

Proposition 2.3. Let $P$ be a measurable order on $\Gamma$. Then $\bar{P}$ is a $\mathcal{T}$-set.

Proof. If $\Gamma$ is discrete, there is nothing to prove. If $\Gamma$ is not discrete, the subgroup $C_{\alpha_{0}}$ is open and nonempty. Hence the set $C_{\alpha_{0}} \cap\{\chi \in \Gamma$ : $\left.\psi_{\alpha_{0}}(\chi)>0\right\}$ is nonempty, with 0 as a limit point. Given an open nonempty neighborhood $U$ of 0 , let

$$
W=U \cap C_{\alpha_{0}} \cap\left\{\chi \in \Gamma: \psi_{\alpha_{0}}(\chi)>0\right\} .
$$

Then $W$ is a nonempty subset of $U \cap P$. Moreover, it is easy to see that $W+\bar{P} \subset P \subset \bar{P}$, and hence $\bar{P}$ is a $\mathcal{T}$-set.

3. Analyticity. We continue with the notation of the previous section. Using the order structure on $\Gamma$ we define some classes of analytic functions on $G$ :

$$
\begin{aligned}
& H^{1}(G)=\left\{f \in L^{1}(G): \widehat{f}=0 \text { on }(-P) \backslash\{0\}\right\}, \\
& H_{0}^{1}(G)=\left\{f \in L^{1}(G): \widehat{f}=0 \text { on }-P\right\}
\end{aligned}
$$

and

$$
H^{\infty}(G)=\left\{f \in L^{\infty}(G): \int_{G} f(x) g(x) d x=0 \text { for all } g \in H_{0}^{1}(G)\right\} .
$$

We clearly have

$$
H^{1}(G)=\left\{f \in L^{1}(G): \widehat{f}=0 \text { on } \overline{(-P) \backslash\{0\}}\right\} .
$$

We can now give the definition of analytic measures in $\mathcal{M}_{T}(\Sigma)$. 
Definition 3.1. Let $T$ be a sup path attaining representation of $G$ by isomorphisms of $M(\Sigma)$. A measure $\mu \in \mathcal{M}_{T}(\Sigma)$ is called weakly analytic if the mapping $t \mapsto T_{t} \mu(A)$ is in $H^{\infty}(G)$ for every $A \in \Sigma$.

Definition 3.2. Recall the T-spectrum of a weakly measurable $\mu \in$ $\mathcal{M}_{T}(\Sigma)$

$$
\operatorname{spec}_{T} \mu=\bigcap_{f \in \mathcal{I}(\mu)}\{\chi \in \Gamma: \widehat{f}(\chi)=0\} .
$$

A measure $\mu$ in $\mathcal{M}_{T}(\Sigma)$ is called T-analytic if $\operatorname{spec}_{T} \mu \subset \bar{P}$.

That the two definitions of analyticity are equivalent will be shown later in this section.

Since $\mathcal{I}(\mu)$ is translation-invariant, it follows readily that for all $t \in G$,

$$
\mathcal{I}\left(T_{t} \mu\right)=\mathcal{I}(\mu)
$$

and hence

$$
\operatorname{spec}_{T} T_{t}(\mu)=\operatorname{spec}_{T} \mu .
$$

We now recall several basic results from spectral theory of bounded functions that will be needed in what follows. Our reference is [20, Section 40]. If $\phi$ is in $L^{\infty}(G)$, write $[\phi]$ for the smallest weak-* closed translation-invariant subspace of $L^{\infty}(G)$ containing $\phi$, and let $\mathcal{I}([\phi])=\mathcal{I}(\phi)$ denote the closed translation-invariant ideal in $L^{1}(G)$ :

$$
\mathcal{I}(\phi)=\left\{f \in L^{1}(G): f * \phi=0\right\} .
$$

It is clear that $\mathcal{I}(\phi)=\left\{f \in L^{1}(G): f * g=0, \forall g \in[\phi]\right\}$. The spectrum of $\phi$, denoted by $\sigma[\phi]$, is the set of all continuous characters of $G$ that belong to $[\phi]$. This closed subset of $\Gamma$ is also given by

$$
\sigma[\phi]=Z(\mathcal{I}(\phi))
$$

(See [20, Theorem (40.5)].)

Recall that a closed subset $E$ of $\Gamma$ is a set of spectral synthesis for $L^{1}(G)$, or an $S$-set, if and only if $\mathcal{I}([E])$ is the only ideal in $L^{1}(G)$ whose zero set is $E$.

There are various equivalent definitions of $S$-sets. Here is one that we will use at several occasions.

A set $E \subset \Gamma$ is an $S$-set if every essentially bounded function $g$ in $L^{\infty}(G)$ with $\sigma[g] \subset E$ is the weak-* limit of linear combinations of characters from $E$.

(See $[20,(40.23)(a)]$.$) This has the following immediate consequence.$

Proposition 3.3. Suppose that $B$ is an $S$-set, $g \in L^{\infty}(G)$, and $\operatorname{spec}(g)$ $\subset B$. 
(i) If $f$ is in $L^{1}(G)$ and $\widehat{f}=0$ on $B$, then $f * g(x)=0$ for all $x$ in $G$. In particular,

$$
\int_{G} f(x) g(-x) d x=0 .
$$

(ii) If $\mu$ is a measure in $M(G)$ with $\widehat{\mu}=0$ on $B$, then $\mu * g(x)=0$ for almost all $x$ in $G$.

Proof. Part (i) is a simple consequence of [20, Theorems (40.8) and (40.10)]. We give a proof for the sake of completeness. Write $g$ as the weak-* limit of trigonometric polynomials, $\sum_{\chi \in E} a_{\chi} \chi(x)$, with characters in $E$. Then

$$
\begin{aligned}
\int_{G} f(x) g(y-x) d x & =\lim \int_{G} \sum_{\chi \in E} a_{\chi} \chi(y) f(x) \chi(-x) d x \\
& =\lim \sum_{\chi \in E} a_{\chi} \chi(y) \widehat{f}(\chi)=0
\end{aligned}
$$

since $\widehat{f}$ vanishes on $E$.

To prove (ii), assume that $\mu * g$ is not 0 a.e. Then there is $f$ in $L^{1}(G)$ such that $f *(\mu * g)$ is not 0 a.e. But this contradicts (i), since $f *(\mu * g)=(f * \mu) * g$, $f * \mu$ is in $L^{1}(G)$, and $\widehat{f * \mu}=0$ on $B$.

The following is a converse of sorts of Proposition 3.3 and follows easily from definitions.

Proposition 3.4. Let $B$ be a nonvoid closed subset of $\Gamma$. Suppose that $f$ is in $L^{\infty}(G)$ and

$$
\int_{G} f(x) g(x) d x=0
$$

for all $g$ in $L^{1}(G)$ such that $\widehat{g}=0$ on $-B$. Then $\sigma[f] \subset B$.

Proof. Let $\chi_{0}$ be any element in $\Gamma \backslash B$. We will show that $\chi_{0}$ is not in the spectrum of $f$ by constructing a function $h$ in $L^{1}(G)$ with $\widehat{h}\left(\chi_{0}\right) \neq 0$ and $h * f=0$. Let $U$ be an open neighborhood of $\chi_{0}$ not intersecting $B$, and let $h$ be in $L^{1}(G)$ such that $\widehat{h}$ is equal to 1 at $\chi_{0}$ and to 0 outside $U$. Direct computations show that the Fourier transform of the function $g: t \mapsto$ $h(x-t)$, when evaluated at $\chi \in \Gamma$, gives $\overline{\chi(x)} \widehat{h}(-\chi)$, and hence it vanishes on $-B$. It follows from (20) that $h * f=0$, which completes the proof.

A certain class of $S$-sets, known as the Calderón sets, or $C$-sets, is particularly useful to us. These are defined as follows. A subset $E$ of $\Gamma$ is called a $C$-set if every $f$ in $L^{1}(G)$ with Fourier transform vanishing on $E$ can be approximated in the $L^{1}$-norm by functions of the form $h * f$ where $h \in L^{1}(G)$ and $\widehat{h}$ vanishes on an open set containing $E$. 

$7.5])$.

$C$-sets enjoy the following properties (see $[20,(39.39)]$ or $[22$, Section

- Every $C$-set is an $S$-set.

- Every closed subgroup of $\Gamma$ is a $C$-set.

- The empty set is a $C$-set.

- If the boundary of a set $A$ is a $C$-set, then $A$ is a $C$-set.

- Finite unions of $C$-sets are $C$-sets.

Since closed subgroups are $C$-sets, we conclude that $\bar{P} \cap \overline{(-P)}$ and $C_{\alpha}$, for all $\alpha$, are $C$-sets. From the definition of $S_{\alpha_{0}},(13)$, and the fact that $C_{\alpha_{0}}$ is open and closed, it follows that the boundary of $S_{\alpha_{0}}$ is the closed subgroup $\psi_{\alpha_{0}}^{-1}(0) \cap C_{\alpha_{0}}$. Hence $S_{\alpha_{0}}$ is a $C$-set. For $\alpha \neq \alpha_{0}$, the set $S_{\alpha}$ is open and closed, and so it has empty boundary, and thus it is a $C$-set. Likewise, $C_{\alpha} \backslash D_{\alpha}$ is a $C$-set for all $\alpha \neq \alpha_{0}$. From this we conclude that arbitrary unions of $S_{\alpha}$ and $C_{\alpha} \backslash D_{\alpha}$ are $C$-sets, because an arbitrary union of such sets, not including the index $\alpha_{0}$, is open and closed, and so it is a $C$-set.

We summarize our findings as follows.

Proposition 3.5. Suppose that $P$ is a measurable order on $\Gamma$. We have:

(i) $\bar{P}$ and $\overline{(-P)}$ are $C$-sets;

(ii) $S_{\alpha}$ is a $C$-set for all $\alpha$;

(iii) arbitrary unions of $S_{\alpha}$ and $C_{\alpha} \backslash D_{\alpha}$ are $C$-sets.

As an immediate application, we have the following characterizations.

Corollary 3.6. Suppose that $f$ is in $L^{\infty}(G)$. Then:

(i) $\sigma[f] \subset S_{\alpha}$ if and only if $\int_{G} f(x) g(x) d x=0$ for all $g \in L^{1}(G)$ such that $\widehat{g}=0$ on $-S_{\alpha}$;

(ii) $\sigma[f] \subset \Gamma \backslash C_{\alpha}$ if and only if $\mu_{\alpha} * f=0$;

(iii) $\sigma[f] \subset \bar{P}$ if and only if $f \in H^{\infty}(G)$.

Proof. Assertions (i) and (iii) are clear from Propositions 3.5 and 3.4. To prove (ii), use Fubini's Theorem to first establish that for any $g \in L^{1}(G)$, and any $\mu \in M(G)$, we have

$$
\int_{G}(\mu * f)(t) g(t) d t=\int_{G} f(t)(\mu * g)(t) d t .
$$

Now suppose that $\sigma[f] \subset \Gamma \backslash C_{\alpha}$, and let $g$ be any function in $L^{1}(G)$. From Propositions 3.5 and 3.4, we have $\int_{G} f g d t=0$ for all $g$ with Fourier transform vanishing on $\Gamma \backslash C_{\alpha}$, equivalently, for all $g=\mu_{\alpha} * g$. Hence, $\int_{G} f\left(\mu_{\alpha} * g\right) d t=\int_{G}\left(\mu_{\alpha} * f\right) g d t=0$ for all $g$ in $L^{1}(G)$, from which it follows that $\mu_{\alpha} * f=0$. The converse is proved similarly, and we omit the details.

Aiming for a characterization of weakly analytic measures in terms of their spectra, we present one more result. 
Proposition 3.7. Let $\mu$ be weakly measurable in $M(\Sigma)$.

(i) Suppose that $B$ is a nonvoid closed subset of $\Gamma$ and $\operatorname{spec}_{T} \mu \subset B$. Then $\sigma\left[t \mapsto T_{t} \mu(A)\right] \subset B$ for all $A \in \Sigma$.

(ii) Conversely, suppose that $B$ is an $S$-set in $\Gamma$ and that $\sigma\left[t \mapsto T_{t} \mu(A)\right]$ $\subset B$ for all $A \in \Sigma$, then $\operatorname{spec}_{T} \mu \subset B$.

Proof. We clearly have $\mathcal{I}(\mu) \subset \mathcal{I}\left(\left[t \mapsto T_{t} \mu(A)\right]\right)$. Hence, $\operatorname{spec}_{T} \mu=$ $Z(\mathcal{I}(\mu)) \supset Z\left(\mathcal{I}\left(\left[t \mapsto T_{t} \mu(A)\right]\right)\right)=\sigma\left[t \mapsto T_{t} \mu(A)\right]$, and (i) follows.

Now suppose that $B$ is an $S$-set and let $g \in L^{1}(G)$ be such that $\widehat{g}=0$ on $-B$. Then, for all $A \in \Sigma$, from Proposition 3.4 we have

$$
\int_{G} g(t) T_{t} \mu(A) d t=0 .
$$

Equivalently,

$$
\int_{G} g(-t) T_{-t} \mu(A) d t=0 .
$$

Since the Fourier transform of the function $t \mapsto g(-t)$ vanishes on $B$, we see that $\mathcal{I}(\mu) \supset\{f: \widehat{f}=0$ on $B\}$. Thus $Z(\mathcal{I}(\mu)) \subset Z(\{f: \widehat{f}=0$ on $B\})=B$, which completes the proof.

Straightforward applications of Propositions 3.5 and 3.7 yield the desired characterization of weakly analytic measures.

Corollary 3.8. Suppose that $\mu \in \mathcal{M}_{T}(\Sigma)$. Then:

(i) $\mu$ is weakly T-analytic if and only if $\operatorname{spec}_{T} \mu \subset \bar{P}$ if and only if $\sigma\left[t \mapsto T_{t} \mu(A)\right] \subset \bar{P}$ for every $A \in \Sigma$;

(ii) $\operatorname{spec}_{T} \mu \subset S_{\alpha}$ if and only if $\sigma\left[t \mapsto T_{t} \mu(A)\right] \subset S_{\alpha}$ for every $A \in \Sigma$.

(iii) $\operatorname{spec}_{T} \mu \subset C_{\alpha}$ if and only if $\sigma\left[t \mapsto T_{t} \mu(A)\right] \subset C_{\alpha}$ for every $A \in \Sigma$.

(iv) $\operatorname{spec}_{T} \mu \subset \Gamma \backslash C_{\alpha}$ if and only if $\sigma\left[t \mapsto T_{t} \mu(A)\right] \subset \Gamma \backslash C_{\alpha}$ for every $A \in \Sigma$.

The remaining results of this section are simple properties of measures in $\mathcal{M}_{T}(\Sigma)$ that will be needed later. Although the statements are direct analogues of classical facts about measures on groups, these generalizations require in some places the sup path attaining property of $T$.

Proposition 3.9. Suppose that $\mu \in \mathcal{M}_{T}(\Sigma)$ and $\nu \in M(G)$. Then $\operatorname{spec}_{T} \nu *_{T} \mu$ is contained in the support of $\widehat{\nu}$, and $\operatorname{spec}_{T} \nu *_{T} \mu \subset \operatorname{spec}_{T} \mu$.

Proof. Given $\chi_{0}$ not in the support of $\widehat{\nu}$, to conclude that it is also not in the spectrum of $\nu *_{T} \mu$ it is enough to find a function $f$ in $L^{1}(G)$ with $\widehat{f}\left(\chi_{0}\right)=1$ and $f *_{T}\left(\nu *_{T} \mu\right)=0$. Simply choose $f$ with Fourier transform vanishing on the support of $\widehat{\nu}$ and taking value 1 at $\chi_{0}$. By Fourier inversion, we have $f * \nu=0$, and since $f *_{T}\left(\nu *_{T} \mu\right)=\left(f *_{\nu}\right) *_{T} \mu$, the first part of the 
proposition follows. For the second part, we have $\mathcal{I}(\mu) \subset \mathcal{I}\left(\nu *_{T} \mu\right)$, which implies the desired inclusion.

We next prove a property of $L^{\infty}(G)$ functions similar to the characterization of $L^{1}$ functions which are constant on cosets of a subgroup [20, Theorem (28.55)].

Proposition 3.10. Suppose that $f$ is in $L^{\infty}(G)$ and that $\Lambda$ is an open subgroup of $\Gamma$. Let $\lambda_{0}$ denote the normalized Haar measure on the compact group $A(G, \Lambda)$, the annihilator in $G$ of $\Lambda$ (see $[19,(23.23)])$. Then $\sigma[f] \subset \Lambda$ if and only if $f=f * \lambda_{0}$ a.e. This is also the case if and only if $f$ is constant on cosets of $A(G, \Lambda)$.

Proof. Suppose that the spectrum of $f$ is contained in $\Lambda$. Since $\Lambda$ is an $S$-set, it follows that $f$ is the weak-* limit of trigonometric polynomials with spectra contained in $\Lambda$. Let $\left\{f_{\alpha}\right\}$ be a net of such trigonometric polynomials converging to $f$ weak- $*$. Note that, for any $\alpha$, we have $\lambda_{0} * f_{\alpha}=f_{\alpha}$. For $g$ in $L^{1}(G)$, we have

$$
\lim _{\alpha} \int_{G} f_{\alpha} \bar{g} d x=\int_{G} f \bar{g} d x
$$

In particular, we have

$$
\lim _{\alpha} \int_{G} f_{\alpha}\left(\lambda_{0} * \bar{g}\right) d x=\int_{G} f\left(\lambda_{0} * \bar{g}\right) d x,
$$

and so

$$
\lim _{\alpha} \int_{G}\left(f_{\alpha} * \lambda_{0}\right) \bar{g} d x=\int_{G}\left(f * \lambda_{0}\right) \bar{g} d x .
$$

Since this holds for any $g$ in $L^{1}(G)$, we conclude that $\lambda_{0} * f_{\alpha}$ converges weak- $^{*}$ to $\lambda_{0} * f$. But $\lambda_{0} * f_{\alpha}=f_{\alpha}$, and $f_{\alpha}$ converges weak- ${ }^{*}$ to $f$, hence $f * \lambda_{0}=f$. The remaining assertions of the lemma are easy to prove. We omit the details.

In what follows, we use the symbol $\mu_{\alpha}$ to denote the normalized Haar measure on the compact subgroup $A\left(G, C_{\alpha}\right)$, the annihilator in $G$ of $C_{\alpha}$. This measure is also characterized by its Fourier transform:

$$
\widehat{\mu}_{\alpha}=1_{C_{\alpha}}
$$

(see $[19,(23.19)])$.

Corollary 3.11. Suppose that $\mu \in \mathcal{M}_{T}(\Sigma)$. Then:

(i) $\operatorname{spec}_{T} \mu \subset C_{\alpha}$ if and only if $\mu=\mu_{\alpha} *_{T} \mu$;

(ii) $\operatorname{spec}_{T} \mu \subset \Gamma \backslash C_{\alpha}$ if and only if $\mu_{\alpha} *_{T} \mu=0$.

Proof. (i) If $\mu=\mu_{\alpha} *_{T} \mu$, then $\sigma\left[t \mapsto \mu_{\alpha} *_{T} T_{t} \mu(A)\right] \subset C_{\alpha}$ by Proposition 3.10. Hence by Corollary 3.8, $\operatorname{spec}_{T} \mu \subset C_{\alpha}$. For the other direction, suppose that $\operatorname{spec}_{T} \mu \subset C_{\alpha}$. Then by Corollary 3.8 the spectrum of the function 
$t \mapsto T_{t} \mu(A)$ is contained in $C_{\alpha}$ for every $A \in \Sigma$. By Proposition 3.10, we have

$$
T_{t} \mu(A)=\int_{G_{\alpha}} T_{t-y} \mu(A) d \mu_{\alpha}=T_{t}\left(\mu_{\alpha} * \mu\right)(A)
$$

for almost all $t \in G$. Since this holds for all $A \in \Sigma$, the desired conclusion follows from Proposition 1.4.

Part (ii) follows from Corollary 3.6(ii), Proposition 3.7(ii), and the fact that $\Gamma \backslash C_{\alpha}$ is an $S$-set.

Corollary 3.12. Suppose that $\mu \in \mathcal{M}_{T}(\Sigma)$ and $\operatorname{spec}_{T} \mu \subset C_{\alpha}$, and let $y \in G_{\alpha}=A\left(G, C_{\alpha}\right)$. Then $T_{y} \mu=\mu$.

Proof. For any $A \in \Sigma$, from Corollary 3.11 we have

$$
\begin{aligned}
T_{y} \mu(A) & =T_{y}\left(\mu_{\alpha} * \mu\right)(A)=\mu_{\alpha} * T_{y} \mu(A)=\int_{G_{\alpha}} T_{y-x} \mu(A) d \mu_{\alpha}(y) \\
& =\int_{G_{\alpha}} T_{-x} \mu(A) d \mu_{\alpha}(y)=\mu_{\alpha} * \mu(A)=\mu(A) .
\end{aligned}
$$

4. Homomorphism theorems. We continue with the notation of the previous section: $G$ is a locally compact abelian group, $\Gamma$ is the dual group of $G, P$ is a measurable order on $\Gamma, T$ is a sup path attaining representation of $G$ acting on $M(\Sigma)$. Associated with $P$ is a collection of homomorphisms $\psi_{\alpha}$, as described by Theorem 2.1. Let $\phi_{\alpha}$ denote the adjoint of $\psi_{\alpha}$. Thus, $\phi_{\alpha}$ is a continuous homomorphism of $\mathbb{R}$ into $G$. By composing the representation $T$ with the $\phi_{\alpha}$, we define a new representation $T_{\phi_{\alpha}}$ of $\mathbb{R}$ acting on $M(\Sigma)$ by $t \in \mathbb{R} \mapsto T_{\phi_{\alpha}(t)}$. If $\mu$ in $M(\Sigma)$ is weakly measurable with respect to $T$ then $\mu$ is also weakly measurable with respect to $T_{\phi_{\alpha}}$. We will further suppose that $T_{\phi_{\alpha}}$ is sup path attaining for each $\alpha$. This is the case with the representations of Example 1.7.

Our goal in this section is to relate the notion of analyticity with respect to $T$ to the notion of analyticity with respect to $T_{\phi_{\alpha}}$. More generally, suppose that $G_{1}$ and $G_{2}$ are two locally compact abelian groups with dual groups $\Gamma_{1}$ and $\Gamma_{2}$, respectively. Let $\psi: \Gamma_{1} \rightarrow \Gamma_{2}$ be a continuous homomorphism, and let $\phi: G_{2} \rightarrow G_{1}$ denote its adjoint homomorphism. Suppose $\nu$ is in $M\left(G_{2}\right)$. We define a Borel measure $\Phi(\nu)$ in $M\left(G_{1}\right)$ on the Borel subsets $A$ of $G_{1}$ by

$$
\Phi(\nu)(A)=\int_{G_{2}} 1_{A} \circ \phi(t) d \nu(t)=\int_{G_{1}} 1_{A} d \Phi(\nu),
$$

where $1_{A}$ is the indicator function of $A$. We have $\|\Phi(g)\|_{M\left(G_{1}\right)}=\|\nu\|_{M\left(G_{2}\right)}$ and, for every Borel measurable bounded function $f$ on $G_{1}$, we have

$$
\int_{G_{1}} f d \Phi(\nu)=\int_{G_{2}} f \circ \phi(t) d \nu(t)
$$


In particular, if $f=\chi$, a character in $\Gamma$, then

$$
\begin{aligned}
\widehat{\Phi(\nu)}(\chi) & =\int_{G_{1}} \bar{\chi} d \Phi(\nu)=\int_{G_{2}} \bar{\chi} \circ \phi(t) d \nu(t) \\
& =\int_{G_{2}} \overline{\psi(\chi)}(t) d \nu(t)=\widehat{\nu}(\psi(\chi)),
\end{aligned}
$$

where $\psi$ is the adjoint homomorphism of $\phi$. So,

$$
\widehat{\Phi(\nu)}=\widehat{\nu} \circ \psi \text {. }
$$

Our first result is a very useful fact from spectral synthesis of bounded functions. The proof uses in a crucial way the fact that the representation is sup path attaining, or, more precisely, satisfies the property in Proposition 1.4.

Lemma 4.1. Suppose that $T$ is a sup path attaining representation of $G_{1}$ acting on $M(\Sigma)$ and $\phi$ is a continuous homomorphism of $G_{2}$ into $G_{1}$ such that $T_{\phi}$ is a sup path attaining representation of $G_{2}$. Suppose that $B$ is a nonempty closed $S$-subset of $\Gamma_{1}$ and that $\mu$ is in $M(\Sigma)$ with $\operatorname{spec}_{T} \mu \subset B$. Suppose that $C$ is an $S$-subset of $\Gamma_{2}$ and $\psi(B) \subset C$. Then $\operatorname{spec}_{T_{\phi}} \mu \subset C$.

Proof. Since $C$ is an $S$-subset of $\Gamma_{2}$, it is enough to show that for every $A \in \Sigma, \operatorname{spec}_{T_{\phi}}\left(x \mapsto T_{\phi(x)} \mu(A)\right) \subset C$, by Proposition 3.7. For this purpose, it is enough, by [20, Theorem (40.8)], to show that

$$
g * T_{\phi(\cdot)} \mu(A)=0
$$

for every $g$ in $L^{1}\left(G_{2}\right)$ with $\widehat{g}=0$ on $C$. For $r \in G_{2}$ and $x \in G_{1}$, consider the measure

$$
T_{x}\left(g * T_{\phi} T_{\phi(r)} \mu\right)=g *_{T_{\phi}} T_{x+\phi(r)} \mu .
$$

For $A \in \Sigma$, we have

$$
\begin{aligned}
g *_{T_{\phi}} T_{x+\phi(r)} \mu(A) & =\int_{G} T_{-t+x}\left(T_{\phi(r)} \mu\right)(A) d \Phi(g)(t) \\
& =\stackrel{\Phi}{\Phi}(g) *\left[t \mapsto T_{t}\left(T_{\phi(r)} \mu\right)(A)\right](x)=0
\end{aligned}
$$

for almost all $x \in G_{1}$. To justify the last equality, we appeal to Proposition 3.3 and note that $\widehat{\Phi(g)}=\widehat{g} \circ \psi$ and so $\widehat{\Phi(g)}=0$ on $B \subset \psi^{-1}(C)$. Moreover, $\sigma\left[t \mapsto T_{t}\left(T_{\phi(r)} \mu\right)(A)\right] \subset \operatorname{spec}_{T} \mu \subset B$. Now, using Proposition 1.4 and the fact that, for every $A \in \Sigma$,

$$
T_{x}\left[g * T_{\phi} T_{\phi(r)}\right] \mu(A)=g * T_{\phi} T_{x+\phi(r)} \mu(A)=0
$$

for almost all $x \in G_{1}$, we conclude that the measure $g * T_{\phi} T_{\phi(r)} \mu$ is the zero measure, which completes the proof. 
Given $\mathcal{C}$, a collection of elements in $L^{1}\left(G_{1}\right)$ or $M\left(G_{1}\right)$, let

$$
Z(\mathcal{C})=\bigcap_{\delta \in \mathcal{C}}\{\chi: \widehat{\delta}(\chi)=0\}
$$

This is the same notation for the zero set of an ideal in $L^{1}(G)$ that we introduced in Section 1. Given a set $\mathcal{S}$ of measures in $M\left(G_{2}\right)$, let

$$
\Phi(\mathcal{S})=\{\Phi(\nu): \nu \in \mathcal{S}\} \subset M\left(G_{1}\right) .
$$

Lemma 4.2. In the above notation, if $\mu \in M(\Sigma)$ is weakly measurable, then

$$
Z\left(\Phi\left(\mathcal{I}_{T_{\phi}} \mu\right)\right)=\psi^{-1}\left(Z\left(\mathcal{I}_{T_{\phi}} \mu\right)\right)=\psi^{-1}\left(\operatorname{spec}_{T_{\phi}} \mu\right) .
$$

Proof. It is enough to establish the first equality; the second one follows from definitions. We have

$$
\begin{aligned}
Z\left(\Phi\left(\mathcal{I}_{T_{\phi}} \mu\right)\right) & =\bigcap_{\delta \in \Phi\left(\mathcal{I}_{T_{\phi}}(\mu)\right)}\{\chi \in \Gamma: \widehat{\delta}(\chi)=0\} \\
& =\bigcap_{g \in \mathcal{I}_{T_{\phi}}(\mu)}\{\chi \in \Gamma: \widehat{\Phi(g)}(\chi)=0\} \\
& =\bigcap_{g \in \mathcal{I}_{T_{\phi}}(\mu)}\{\chi \in \Gamma: \widehat{g}(\psi(\chi))=0\} \\
& =\bigcap_{g \in \mathcal{I}_{T_{\phi}}(\mu)} \psi^{-1}(Z(g))=\psi^{-1}\left(\bigcap_{g \in \mathcal{I}_{T_{\phi}}(\mu)} Z(g)\right) \\
& =\psi^{-1}\left(Z\left(\mathcal{I}_{T_{\phi}}(\mu)\right)\right)=\psi^{-1}\left(\operatorname{spec}_{T_{\phi}} \mu\right) .
\end{aligned}
$$

Lemma 4.3. Suppose that $C$ is a nonempty closed $S$-subset of $\Gamma_{2}$ and that $\psi^{-1}(C)$ is an $S$-subset of $\Gamma_{1}$. Suppose that $\mu$ is in $M(\Sigma)$ and $\operatorname{spec}_{T_{\phi}} \mu$ $\subset C$. Then $\operatorname{spec}_{T} \mu \subset \psi^{-1}(C)$.

Proof. We will use the notation of Lemma 4.2. If $f \in \mathcal{I}_{T_{\phi}}(\mu)$ and $t \in G_{1}$, then $f \in \mathcal{I}_{T_{\phi}}\left(T_{t} \mu\right)$. So, for $A \in \Sigma$, we have $f * T_{\phi}\left(T_{t} \mu\right)(A)=0$. But

$$
f * T_{T_{\phi}}\left(T_{t} \mu\right)(A)=\int_{\mathbb{R}} T_{t-\phi(x)} \mu(A) f(x) d x=\int_{G} T_{t-x} \mu(A) d \Phi(f),
$$

where $\Phi(f)$ is the homomorphic image of the measure $f(x) d x$. Hence, $\Phi(f) \in$ $\mathcal{I}_{T}\left(\left[t \mapsto T_{t} \mu(A)\right]\right)$, and so $\Phi\left(\mathcal{I}_{T_{\phi}}(\mu)\right) \subset \mathcal{I}_{T}\left(\left[t \mapsto T_{t} \mu(A)\right]\right)$, which implies that

$$
Z\left(\Phi\left(\mathcal{I}_{T_{\phi}}(\mu)\right)\right) \supset Z\left(\mathcal{I}_{T}\left(\left[t \mapsto T_{t} \mu(A)\right]\right)\right)=\operatorname{spec}_{T}\left(t \mapsto T_{t} \mu(A)\right) .
$$

By Lemma 4.2 ,

$$
Z\left(\Phi\left(\mathcal{I}_{T_{\phi}}(\mu)\right)\right)=\psi^{-1}\left(\operatorname{spec}_{T_{\phi}} \mu\right) \subset \psi^{-1}(C) .
$$

Hence, $\operatorname{spec}_{T}\left(t \mapsto T_{t} \mu(A)\right) \subset \psi^{-1}(C)$ for all $A \in \Sigma$, which by Proposition 3.7 implies that $\operatorname{spec}_{T} \mu \subset \psi^{-1}(C)$. 
Taking $G_{1}=G, G_{2}=\mathbb{R}$ and $\psi=\psi_{\alpha}$ to be one of the homomorphisms in Theorem 2.1, and using the fact that $\left[0, \infty\left[, S_{\alpha}, C_{\alpha} \backslash D_{\alpha}\right.\right.$ are all $S$-sets, we obtain useful relationships between different types of analyticity.

THEOREM 4.4. Let $G$ be a locally compact abelian group with ordered dual group $\Gamma$, and let $P$ denote a measurable order on $\Gamma$. Suppose that $T$ is a sup path attaining representation of $G$ by isomorphisms of $M(\Sigma)$ such that $T_{\phi_{\alpha}}$ is sup path attaining, where $\phi_{\alpha}$ is the adjoint of $\psi_{\alpha}$ in Theorem 2.1.

(i) If $\mu \in M(\Sigma)$ and $\operatorname{spec}_{T} \mu \subset C_{\alpha} \backslash D_{\alpha}$, then

$$
\operatorname{spec}_{T} \mu \subset S_{\alpha} \Leftrightarrow \operatorname{spec}_{T_{\phi_{\alpha}}} \mu \subset[0, \infty[.
$$

(ii) If $\mu \in M(\Sigma)$ and $\operatorname{spec}_{T} \mu \subset C_{\alpha_{0}}$. Then

$$
\operatorname{spec}_{T} \mu \subset S_{\alpha_{0}} \Leftrightarrow \operatorname{spec}_{T_{\phi_{0}}} \mu \subset[0, \infty[\text {. }
$$

We can use the representation $T_{\phi}$ to convolve a measure $\nu \in M\left(G_{2}\right)$ with $\mu \in M\left(G_{1}\right):$

$$
\nu *_{T_{\phi}} \mu(A)=\int_{G_{2}} T_{-\phi(x)} \mu(A) d \nu(x)=\int_{G_{2}} \mu(A-\phi(x)) d \nu(x)
$$

for all Borel $A \subset G_{1}$.

Alternatively, we can convolve $\Phi(\nu)$ in the usual sense of [19, Definition 19.8 ] with $\mu$ to yield another measure in $M\left(G_{1}\right)$, defined on the Borel subsets of $G_{1}$ by

$$
\Phi(\nu) * \mu(A)=\int_{G_{1}} \int_{G_{1}} 1_{A}(x+y) d \Phi(\nu)(x) d \mu(y) .
$$

Using (22), we find that

$$
\begin{aligned}
\Phi(\nu) * \mu(A) & =\iint_{G_{1}} 1_{G_{2}} 1_{A}(\phi(t)+y) d \nu(t) d \mu(y) \\
& =\int_{G_{2}} \mu(A-\phi(t)) d \nu(t)=\nu *_{T_{\phi}} \mu(A) .
\end{aligned}
$$

Thus,

$$
\Phi(\nu) * \mu=\nu * T_{\phi} \mu .
$$

We end the section with homomorphism theorems, which complement the well known homomorphism theorems for $L^{p}$-multipliers (see Edwards and Gaudry [10, Appendix B]). In these theorems, we let $G_{1}$ act on $M\left(G_{1}\right)$ by translation. That is, if $\mu \in M\left(G_{1}\right), x \in G_{1}$, and $A$ is a Borel subset of $G_{1}$, then

$$
T_{x} \mu(A)=\mu(A+x) .
$$

Let $\phi: G_{2} \rightarrow G_{1}$ be a continuous homomorphism. By Example 1.3, $T$ and $T_{\phi}$ are sup path attaining. (Recall that if $t \in G_{2}, \mu \in M\left(G_{1}\right)$, then 
$T_{\phi(t)} \mu(A)=\mu(A+\phi(t))$.) A simple exercise with definitions shows that for $\mu \in M\left(G_{1}\right)$,

$$
\operatorname{spec}_{T} \mu=\operatorname{supp} \widehat{\mu} .
$$

TheOREM 4.5. Suppose that $\Gamma_{1}$ and $\Gamma_{2}$ contain measurable orders $P_{1}$ and $P_{2}$, respectively, and $\psi: \Gamma_{1} \rightarrow \Gamma_{2}$ is a continuous, order-preserving homomorphism (that is, $\left.\psi\left(\bar{P}_{1}\right) \subset \bar{P}_{2}\right)$. Suppose that there is a positive constant $N(\nu)$ such that for all $f \in H^{1}\left(G_{2}\right)$,

$$
\|\nu * f\|_{1} \leq N(\nu)\|f\|_{1} .
$$

Then

$$
\|\Phi(\nu) * \mu\| \leq N(\nu)\|\mu\|
$$

for all Borel measures in $M\left(G_{1}\right)$ such that $\widehat{\mu}$ is supported in $\bar{P}_{1}$.

Proof. We have $\Phi(\nu) * \mu=\nu *_{T_{\phi}} \mu$. Also, $\bar{P}_{2}$ is a $\mathcal{T}$-set. So (27) will follow from Theorem 1.8 once we show that $\operatorname{spec}_{T_{\phi}} \mu \subset \bar{P}_{2}$. For that purpose, we use Lemma 4.1. We have

$$
\operatorname{spec}_{T} \mu=\operatorname{supp} \widehat{\mu} \subset \bar{P}_{1},
$$

and $\psi\left(\bar{P}_{1}\right) \subset \bar{P}_{2}$ is an $S$-set. Hence $\operatorname{spec}_{T_{\phi}} \mu \subset \bar{P}_{2}$ by Lemma 4.1 .

The following special case of Theorem 4.5 deserves a separate statement.

THEOREM 4.6. With the above notation, suppose that there is a positive constant $N(\nu)$ such that for all $f \in H^{1}\left(G_{2}\right)$,

$$
\|\nu * f\|_{1} \leq N(\nu)\|f\|_{1} .
$$

Then for all $f \in H^{1}\left(G_{1}\right)$ we have

$$
\|\Phi(\nu) * f\|_{1} \leq N(\nu)\|f\|_{1} .
$$

THEOREM 4.7. Suppose that there is a positive constant $N(\nu)$ such that for all $f \in H^{1}(\mathbb{R})$,

$$
\|\nu * f\|_{1} \leq N(\nu)\|f\|_{1} .
$$

Then for all $\mu \in M(G)$ with support of $\widehat{\mu}$ contained in $C_{\alpha} \backslash D_{\alpha}$, where $\alpha<\alpha_{0}$, we have

$$
\left\|\Phi_{\alpha}(\nu) * \mu\right\|_{1} \leq N(\nu)\|\mu\| .
$$

Proof. The proof is very much like the proof of Theorem 4.5. We have $\Phi_{\alpha}(\nu) * \mu=\nu *_{T_{\phi}} \mu$. Apply Theorem 1.8, taking into consideration that

$$
\operatorname{spec}_{T} \mu=\operatorname{supp} \widehat{\mu} \subset C_{\alpha} \backslash D_{\alpha}
$$

is an $S$-set and so

$$
\operatorname{spec}_{T_{\phi_{\alpha}}} \mu \subset \psi_{\alpha}\left(C_{\alpha} \backslash D_{\alpha}\right) \subset[0, \infty[.
$$


5. Decomposition of analytic measures. Define measures $\mu_{\alpha_{0}}$ and $d_{\alpha}$ by their Fourier transforms: $\widehat{\mu}_{\alpha_{0}}=1_{C_{\alpha_{0}}}$ and $\widehat{d}_{\alpha}=1_{C_{\alpha} \backslash D_{\alpha}}$. Then we have the following decomposition theorem.

THEOREM 5.1. Let $G$ be a locally compact abelian group with an ordered dual group $\Gamma$. Suppose that $T$ is a sup path attaining representation of $G$ in $M(\Sigma)$. Then for any weakly analytic measure $\mu \in M(\Sigma)$ the set of $\alpha$ for which $d_{\alpha} *_{T} \mu \neq 0$ is countable, and

$$
\mu=\mu_{\alpha_{0}} *_{T} \mu+\sum_{\alpha} d_{\alpha} *_{T} \mu,
$$

where the right side converges unconditionally in norm in $M(\Sigma)$. Furthermore, there is a positive constant $c$, depending only upon $T$, such that for any signs $\varepsilon_{\alpha}= \pm 1$ we have

$$
\left\|\sum_{\alpha} \varepsilon_{\alpha} d_{\alpha} *_{T} \mu\right\| \leq c\|\mu\| .
$$

One should compare this theorem to the well known results from Littlewood-Paley theory on $L^{p}(G)$, where $1<p<\infty$ (see Edwards and Gaudry [10]). For $L^{p}(G)$ with $1<p<\infty$, it is well known that the subgroups $\left(C_{\alpha}\right)$ form a Littlewood-Paley decomposition of the group $\Gamma$, which means that the martingale difference series

$$
f=\mu_{\alpha_{0}} * f+\sum_{\alpha} d_{\alpha} * f
$$

converges unconditionally in $L^{p}(G)$ to $f$. Thus, theorem 5.1 above may be considered as an extension of Littlewood-Paley theory to spaces of analytic measures.

The next result, crucial to our proof of Theorem 5.1, is already known in the case of $G=\mathbb{T}^{n}$ with the lexicographic order on the dual. This is due to Garling [14], and is a modification of the celebrated inequalities of Burkholder. Our result can be obtained directly from the result in [14] by combining the techniques of [3] with the homomorphism theorem 4.5 . However, we shall take a different approach, in effect reproducing Garling's proof in this more general setting.

THEOREM 5.2. Suppose that $G$ is a locally compact group with ordered dual $\Gamma$. Then for $f \in H^{1}(G)$, for any set $\left\{\alpha_{j}\right\}_{j=1}^{n}$ of indices less than $\alpha_{0}$, and for any numbers $\varepsilon_{j} \in\{0, \pm 1\}(1 \leq j \leq n)$, there is an absolute constant $a>0$ such that

$$
\left\|\sum_{j=1}^{n} \varepsilon_{j} d_{\alpha_{j}} * f\right\|_{1} \leq a\|f\|_{1} .
$$


Furthermore,

$$
f=\mu_{\alpha_{0}} * f+\sum_{\alpha} d_{\alpha} * f
$$

where the right hand side converges unconditionally in the norm topology on $H^{1}(G)$.

Proof. The second part of Theorem 5.2 follows easily from the first part and Fourier inversion.

Now let us show that if we have the result for compact $G$, then we have it for locally compact $G$. Let $\pi_{\alpha_{0}}: \Gamma \rightarrow \Gamma / C_{\alpha_{0}}$ denote the quotient homomorphism of $\Gamma$ onto the discrete group $\Gamma / C_{\alpha_{0}}$ (recall that $C_{\alpha_{0}}$ is open), and define a measurable order on $\Gamma / C_{\alpha_{0}}$ to be $\pi_{\alpha_{0}}(P)$. By Remark 2.2(c), the decomposition of the group $\Gamma / C_{\alpha_{0}}$ that we get by applying Theorem 2.1 to that group is precisely the image by $\pi_{\alpha_{0}}$ of the decomposition of the group $\Gamma$. Let $G_{0}$ denote the compact dual group of $\Gamma / C_{\alpha_{0}}$. Thus if Theorem 5.2 holds for $H^{1}\left(G_{0}\right)$, then applying Theorem 4.5, we see that Theorem 5.2 holds for $G$.

Henceforth, let us suppose that $G$ is compact. We will suppose that the Haar measure on $G$ is normalized, so that $G$ with Haar measure is a probability space.

Since each one of the subgroups $C_{\alpha}$ and $D_{\alpha}\left(\alpha<\alpha_{0}\right)$ is open, it follows that their annihilators in $G, G_{\alpha}=A\left(G, C_{\alpha}\right)$ and $A\left(G, D_{\alpha}\right)$, are compact. Let $\mu_{\alpha}$ and $\nu_{\alpha}$ denote the normalized Haar measures on $A\left(G, C_{\alpha}\right)$ and $A\left(G, D_{\alpha}\right)$, respectively. We have $\widehat{\mu}_{\alpha}=1_{C_{\alpha}}$ (for all $\alpha$ ) and $\widehat{\nu}_{\alpha}=1_{D_{\alpha}}\left(\right.$ for all $\left.\alpha \neq \alpha_{0}\right)$, so that $d_{\alpha}=\mu_{\alpha}-\nu_{\alpha}$.

For each $\alpha$, let $\mathcal{B}_{\alpha}$ denote the $\sigma$-algebra of subsets of $G$ of the form $A+G_{\alpha}$, where $A$ is a Borel subset of $G$. We have $\mathcal{B}_{\alpha_{1}} \subset \mathcal{B}_{\alpha_{2}}$ whenever $\alpha_{1}>\alpha_{2}$. It is a simple matter to see that for $f \in L^{1}(G)$, the conditional expectation of $f$ with respect to $\mathcal{B}_{\alpha}$ is equal to $\mu_{\alpha} * f$ (see [10, Chapter 5 , Section 2]).

We may suppose without loss of generality that $\alpha_{1}>\ldots>\alpha_{n}$. Thus the $\sigma$-algebras $\mathcal{B}_{\alpha_{k}}$ form a filtration, and the sequence $\left(d_{\alpha_{1}} * f, \ldots, d_{\alpha_{n}} * f\right)$ is a martingale difference sequence with respect to this filtration.

In that case, we have the following result of Burkholder ([7, Inequality $(1.7)$ ] and [8]). If $0<p<\infty$, then there is a positive constant $c$, depending only upon $p$, such that

$$
\left\|\sup _{1 \leq k \leq n}\left(\sum_{j=1}^{k} \varepsilon_{j} d_{\alpha_{j}} * f\right)\right\|_{p} \leq c\left\|\sup _{1 \leq k \leq n}\left(\sum_{j=1}^{k} d_{\alpha_{j}} * f\right)\right\|_{p} .
$$

Lemma 5.3. For any index $\alpha, 0<p<\infty$, and $f \in H^{1}(G) \cap L^{p}(G)$, we have almost everywhere on $G$,

$$
\left|\mu_{\alpha} * f\right|^{p} \leq \mu_{\alpha} *|f|^{p},
$$


where $\mu_{\alpha}$ is the normalized Haar measure on the compact subgroup $G_{\alpha}=$ $A\left(G, C_{\alpha}\right)$.

Proof. The dual group of $G_{\alpha}$ is $\Gamma / C_{\alpha}$ and can be ordered by the set $\pi_{\alpha}(P)$, where $\pi_{\alpha}$ is the natural homomorphism of $\Gamma$ onto $\Gamma / C_{\alpha}$.

Next, by convolving with an approximate identity for $L^{1}(G)$ consisting of trigonometric polynomials, we may assume that $f$ is a trigonometric polynomial. Then we see that for each $x \in G$ the function $y \mapsto f(x+y), y \in G_{\alpha}$, is in $H^{1}\left(G_{\alpha}\right)$. To verify this, it is sufficient to consider the case when $f$ is a character in $P$. Then

$$
f(x+y)=f(x) \pi_{\alpha}(f)(y),
$$

and by definition $\pi_{\alpha}(f)$ is in $H^{1}\left(G_{\alpha}\right)$.

Now we have the following generalization of Jensen's inequality, due to Helson and Lowdenslager [15, Theorem 2]. An independent proof based on the ideas of this section is given in [3]. For all $g \in H^{1}(G)$,

$$
\left|\int_{G} g(x) d x\right| \leq \exp \int_{G} \log |g(x)| d x .
$$

Apply (38) to $y \mapsto f(x+y), y \in G_{\alpha}$, to obtain

$$
\left|\int_{G_{\alpha}} f(x+y) d \mu_{\alpha}(y)\right| \leq \exp \int_{G_{\alpha}} \log |f(x+y)| d \mu_{\alpha}(y) .
$$

Extending the integrals to the whole of $G$ (since $\mu_{\alpha}$ is supported on $G_{\alpha}$ ), raising both sides to the $p$ th power, and then applying the usual Jensen inequality for the logarithmic function on finite measure spaces, we obtain

$$
\left|\int_{G} f(x+y) d \mu_{\alpha}(y)\right|^{p} \leq \exp \int_{G} \log |f(x+y)|^{p} d \mu_{\alpha}(y) \leq \int_{G}|f(x+y)|^{p} d \mu_{\alpha}(y) .
$$

Changing $y$ to $-y$, we obtain the desired inequality.

Let us continue with the proof of Theorem 5.2. We may suppose that $f$ is a mean zero trigonometric polynomial, and that the spectrum of $f$ is contained in $\bigcup_{j=1}^{n} C_{\alpha_{j}} \backslash D_{\alpha_{j}}$, that is to say,

$$
f=\sum_{j=1}^{n} d_{\alpha_{j}} * f .
$$

By Lemma 5.3, we have

$$
\sup _{1 \leq k \leq n}\left|\mu_{\alpha_{k}} * f\right|=\left(\sup _{1 \leq k \leq n}\left|\mu_{\alpha_{k}} * f\right|^{1 / 2}\right)^{2} \leq\left(\sup _{1 \leq k \leq n} \mu_{\alpha_{k}} *|f|^{1 / 2}\right)^{2} .
$$

Also, $\left(\mu_{\alpha_{j}} *|f|^{1 / 2}\right)_{j=1}^{n}$ is a martingale with respect to the filtration $\left(\mathcal{B}_{j}\right)_{j=1}^{n}$. Hence, by Doob's maximal inequality [9, Theorem (3.1), p. 317] we have

$$
\left\|\sup _{1 \leq k \leq n^{\prime}} \mu_{\beta_{k}} *|f|^{1 / 2}\right\|_{2}^{2} \leq 4\left\|\mu_{\beta_{n^{\prime}}} *|f|^{1 / 2}\right\|_{2}^{2} \leq 4\left\||f|^{1 / 2}\right\|_{2}^{2}=4\|f\|_{1}
$$


The desired inequality now follows upon combining Burkholder's inequality (36) with (39), and (40).

Proof of Theorem 5.1. Transferring inequality (34) by using Theorem 1.8 , we deduce that for any set $\left\{\alpha_{j}\right\}_{j=1}^{n}$ of indices less than $\alpha_{0}$, and for any numbers $\varepsilon_{j} \in\{0, \pm 1\}(1 \leq j \leq n)$, there is a positive constant $c$, depending only upon the representation $T$, such that

$$
\left\|\sum_{j=1}^{n} \varepsilon_{j} d_{\alpha_{j}} *_{T} \mu\right\| \leq c\|\mu\| .
$$

Now suppose that $\left\{\alpha_{j}\right\}_{j=1}^{\infty}$ is a countable collection of indices less than $\alpha_{0}$. Then by Bessaga and Pełczyński [5], the series $\sum_{j=1}^{\infty} d_{\alpha_{j}} *_{T} \mu$ is unconditionally convergent. In particular, for any $\delta>0$, for only finitely many $k$ do we have $\left\|d_{\alpha_{k}} *_{T} \mu\right\|>\delta$. Since this is true for all such countable sets, we deduce that the set of $\alpha$ for which $d_{\alpha} *_{T} \mu \neq 0$ is countable.

Hence $\sum_{\alpha} d_{\alpha} *_{T} \mu$ is unconditionally convergent to some measure, say $\nu$. Clearly, $\nu$ is weakly measurable. To prove that $\mu=\nu$, it is enough by Proposition 1.4 to show that for every $A \in \Sigma$, we have $T_{t} \mu(A)=T_{t} \nu(A)$ for almost all $t \in G$.

We first note that since for every $f \in L^{1}(G)$ the series $\mu_{\alpha_{0}} * f+\sum_{\alpha} d_{\alpha} * f$ converges to $f$ in $L^{1}(G)$, it follows that, for every $g \in L^{\infty}(G)$, the series $\mu_{\alpha_{0}} *$ $g+\sum_{\alpha} d_{\alpha} * g$ converges to $g$ in the weak-* topology of $L^{\infty}(G)$. Now, on the one hand, for $t \in G$ and $A \in \Sigma$, we have $\mu_{\alpha_{0} *_{T}} T_{t} \mu(A)+\sum_{\alpha} d_{\alpha} *_{T} T_{t} \mu(A)=$ $T_{t} \nu(A)$, because of the (unconditional) convergence of the series $\mu_{\alpha_{0}} *_{T} \mu+$ $\sum_{\alpha} d_{\alpha} *_{T} \mu$ to $\nu$. On the other hand, by considering the $L^{\infty}(G)$ function $t \mapsto T_{t}(A)$, we have $\mu_{\alpha_{0}} *_{T} T_{t} \mu(A)+\sum_{\alpha} d_{\alpha} *_{T} T_{t} \mu(A)=\mu_{\alpha_{0}} * T_{t} \mu(A)+$ $\sum_{\alpha} d_{\alpha} * T_{t} \mu(A)=T_{t} \mu(A)$, weak-*. Thus $T_{t} \mu(A)=T_{t} \nu(A)$ for almost all $t \in G$, and the proof is complete.

6. Generalized F. and M. Riesz theorems. Throughout this section, we adopt the notation of Section 5, specifically, the notation and assumptions of Theorem 5.1.

Suppose that $T$ is a sup path attaining representation of $\mathbb{R}$ by isomorphisms of $M(\Sigma)$. In [4], we proved the following result concerning bounded operators $\mathcal{P}$ from $M(\Sigma)$ into $M(\Sigma)$ that commute with the representation $T$ in the following sense:

$$
\mathcal{P} \circ T_{t}=T_{t} \circ \mathcal{P}
$$

for all $t \in \mathbb{R}$.

THEOREM 6.1. Suppose that $T$ is a representation of $\mathbb{R}$ that is sup path attaining, and that $\mathcal{P}$ commutes with $T$. Let $\mu \in M(\Sigma)$ be weakly analytic. Then $\mathcal{P} \mu$ is also weakly analytic. 
To describe an interesting application of this theorem from [4], let us recall the following.

Definition 6.2. Let $T$ be a sup path attaining representation of $G$ in $M(\Sigma)$. A weakly measurable $\sigma$ in $M(\Sigma)$ is called quasi-invariant if $T_{t} \sigma$ and $\sigma$ are mutually absolutely continuous for all $t \in G$. Hence if $\sigma$ is quasi-invariant and $A \in \Sigma$, then $|\sigma|(A)=0$ if and only if $\left|T_{t}(\sigma)\right|(A)=0$ for all $t \in G$.

Using Theorem 6.1 we obtained in [4] the following extension of results of de Leeuw-Glicksberg [21] and Forelli [11], concerning quasi-invariant measures.

THEOREM 6.3. Suppose that $T$ is a sup path attaining representation of $\mathbb{R}$ by isometries of $M(\Sigma)$. Suppose that $\mu \in M(\Sigma)$ is weakly analytic, and $\sigma$ is quasi-invariant. Write $\mu=\mu_{\mathrm{a}}+\mu_{\mathrm{s}}$ for the Lebesgue decomposition of $\mu$ with respect to $\sigma$. Then both $\mu_{\mathrm{a}}$ and $\mu_{\mathrm{s}}$ are weakly analytic. In particular, the spectra of $\mu_{\mathrm{a}}$ and $\mu_{\mathrm{s}}$ are contained in $[0, \infty)$.

Our goal in this section is to extend Theorems 6.1 above to representations of a locally compact abelian group $G$ with ordered dual group $\Gamma$. More specifically, we want to prove the following theorems.

THEOREM 6.4. Suppose that $T$ is a sup path attaining representation of $G$ by isomorphisms of $M(\Sigma)$ such that $T_{\phi_{\alpha}}$ is sup path attaining for each $\alpha$. Suppose that $\mathcal{P}$ commutes with $T$ in the sense that

$$
\mathcal{P} \circ T_{t}=T_{t} \circ \mathcal{P}
$$

for all $t \in G$. Let $\mu \in M(\Sigma)$ be weakly analytic. Then $\mathcal{P} \mu$ is also weakly analytic.

As shown in $[4$, Theorem (4.10)] for the case $G=\mathbb{R}$, an immediate corollary of Theorem 6.4 is the following result.

THEOREM 6.5. Suppose that $T$ is a sup path attaining representation of $G$ by isometries of $M(\Sigma)$ such that $T_{\phi_{\alpha}}$ is sup path attaining for each $\alpha$. Suppose that $\mu \in M(\Sigma)$ is weakly analytic with respect to $T$, and $\sigma$ is quasiinvariant with respect to $T$. Write $\mu=\mu_{\mathrm{a}}+\mu_{\mathrm{s}}$ for the Lebesgue decomposition of $\mu$ with respect to $\sigma$. Then both $\mu_{\mathrm{a}}$ and $\mu_{\mathrm{s}}$ are weakly analytic with respect to $T$. In particular, the T-spectra of $\mu_{\mathrm{a}}$ and $\mu_{\mathrm{s}}$ are contained in $\bar{P}$.

Proof of Theorem 6.4. Write

$$
\mu=\mu_{\alpha_{0}} *_{T} \mu+\sum_{\alpha} d_{\alpha} *_{T} \mu,
$$

as in (5.1), where the series converges unconditionally in $M(\Sigma)$. Then

$$
\mathcal{P} \mu=\mathcal{P}\left(\mu_{\alpha_{0}} *_{T} \mu\right)+\sum_{\alpha} \mathcal{P}\left(d_{\alpha} *_{T} \mu\right) .
$$


It is enough to show that the $T$-spectrum of each term is contained in $\bar{P}$. Consider the measure $\mu_{\alpha_{0}} *_{T} \mu$. We have $\operatorname{spec}_{T}\left(\mu_{\alpha_{0}} *_{T} \mu\right) \subset S_{\alpha_{0}}$. Hence by Theorem 4.4, $\mu_{\alpha_{0}} *_{T} \mu$ is $T_{\phi_{\alpha_{0}}}$-analytic. Applying Theorem 6.1, we see that

$$
\operatorname{spec}_{T_{\phi_{\alpha_{0}}}} \mathcal{P}\left(\mu_{\alpha_{0}} *_{T} \mu\right) \subset[0, \infty[.
$$

Since $\mathcal{P}$ commutes with $T$, it is easy to see from Proposition 3.10 and Corollary 3.11 that

$$
\operatorname{spec}_{T} \mathcal{P}\left(\mu_{\alpha_{0}} *_{T} \mu\right) \subset C_{\alpha_{0}} .
$$

Hence by (43) and Theorem 4.4,

$$
\operatorname{spec}_{T} \mathcal{P}\left(\mu_{\alpha_{0}} *_{T} \mu\right) \subset S_{\alpha_{0}},
$$

which shows the desired result for the first term of that series in (42). The other terms of that series are handled similarly.

Acknowledgments. The second author is grateful for financial support from the National Science Foundation (U.S.A.) and the Research Board of the University of Missouri.

\section{References}

[1] N. Asmar and S. Montgomery-Smith, Hahn's embedding theorem for orders and harmonic analysis on groups with ordered duals, Colloq. Math. 70 (1996), 235-252.

[2] —, - Analytic measures and Bochner measurability, Bull. Sci. Math. 122 (1998), $39-66$.

[3] - - - Hardy martingales and Jensen's inequality, Bull. Austral. Math. Soc. 55 (1997), 185-195.

[4] N. Asmar, S. Montgomery-Smith and S. Saeki, Transference in spaces of measures, J. Funct. Anal. 165 (1999), 1-23.

[5] C. Bessaga and A. Pełczyński, On bases and unconditional convergence of series in Banach spaces, Studia Math. 17 (1958), 151-164.

[6] S. Bochner, Boundary values of analytic functions in several variables and almost periodic functions, Ann. of Math. 45 (1944), 708-722.

[7] D. L. Burkholder, A geometrical characterization of Banach spaces in which martingale difference sequences are unconditional, Ann. Math. Statist. 37 (1966), 14941504.

[8] —, Martingale transforms, Ann. Probab. 9 (1981), 997-1011.

[9] J. L. Doob, Stochastic Processes, Wiley Publications in Statistics, Wiley, New York, 1953.

[10] R. E. Edwards and G. I. Gaudry, Littlewood-Paley and Multiplier Theory, Ergeb. Math. Grenzgeb. 90, Springer, Berlin, 1977.

[11] F. Forelli, Analytic and quasi-invariant measures, Acta Math. 118 (1967), 33-59.

[12] L. Fuchs, Partially Ordered Algebraic Systems, Pergamon Press, Oxford, 1960.

[13] D. J. H. Garling, On martingales with values in a complex Banach space, Math. Proc. Cambridge Philos. Soc. 104 (1988), 399-406.

[14] - Hardy martingales and the unconditional convergence of martingales, Bull. London Math. Soc. 23 (1991), 190-192. 
[15] H. Helson and D. Lowdenslager, Prediction theory and Fourier series in several variables, Acta Math. 99 (1958), 165-202.

[16] - - - Prediction theory and Fourier series in several variables II, ibid. 106 (1961), $175-212$.

[17] E. Hewitt and S. Koshi, Orderings in locally compact Abelian groups and the theorem of F. and M. Riesz, Math. Proc. Cambridge Philos. Soc. 93 (1983), 441-457.

[18] E. Hewitt, S. Koshi and Y. Takahashi, The F. and M. Riesz Theorem revisited, Math. Scand. 60 (1987), 63-76.

[19] E. Hewitt and K. A. Ross, Abstract Harmonic Analysis I, 2nd ed., Grundlehren Math. Wiss. 115, Springer, Berlin, 1979.

[20] —, - Abstract Harmonic Analysis II, Grundlehren Math. Wiss. 152, Springer, New York, 1970.

[21] K. de Leeuw and I. Glicksberg, Quasi-invariance and analyticity of measures on compact groups, Acta Math. 109 (1963), 179-205.

[22] W. Rudin, Fourier Analysis on Groups, Interscience Tracts in Pure and Appl. Math. 12, Wiley, New York, 1962.

[23] H. Yamaguchi, A property of some Fourier-Stieltjes transforms, Pacific J. Math. 108 (1983), 243-256.

Department of Mathematics

University of Missouri

Columbia, MO 65211, U.S.A.

E-mail: nakhle@math.missouri.edu stephen@math.missouri.edu

Received May 18, 2000

Revised version December 15, 2000 